\title{
A Case of Extra-adrenal Retroperitoneal Ganglioneuroma in a 9-year-old Female: A New Case Report with a Comprehensive Literature Review
}

\author{
Zlatan Zvizdic ${ }^{1}$, Nedzad Rustempasic ${ }^{2}$, Irmina Sefic Pasic ${ }^{3}$, Faruk Skenderi ${ }^{4}$, Semir Vranic ${ }^{5}$ \\ ${ }^{1}$ Clinic of Pediatric Surgery, University Clinical Center Sarajevo, Sarajevo, Bosnia and Herzegovina, ${ }^{2}$ Clinic of Cardiovascular \\ Surgery, University Clinical Center Sarajevo, Sarajevo, Bosnia and Herzegovina, ${ }^{3}$ Department of Radiology, University Clinical \\ Center Sarajevo, Sarajevo, Bosnia and Herzegovina, ${ }^{4}$ Department of Pathology, University Clinical Center Sarajevo, Sarajevo, \\ Bosnia and Herzegovina and ${ }^{5}$ College of Medicine, QU Health, Qatar University, Doha, Qatar
}

Correspondence: semir.vranic@gmail.com or svranic@qu.edu.qa: Tel.: + 9744403 7873; Fax.: + 9744403 3344; ORCID: http://orcid.org/0000-0001-9743-7265

Received: April 25, 2020 Accepted: May 21, 2020

\begin{abstract}
Objective - We present herein a new case and survey comprehensively literature on this rare condition. Case report - A 9-yearold girl with a medical history of surgical correction of clubfoot three months earlier presented to our department with an incidentally detected abdominal mass during diagnostic workup for orthopedic surgery. A CT scan revealed a solid right extra-adrenal mass measuring $7 \times 6 \mathrm{~cm}$. It was compressing/involving the infrahepatic part of inferior vena cava, right renal vein and artery with an incomplete encasing of the abdominal aorta. The surgery was successfully performed. The histopathological analysis confirmed GN. Conclusions - Although pediatric extra-adrenal retroperitoneal ganglioneuroma (GN) are rare, their propensity for enveloping major blood vessels is not. GNs should be considered in the differential diagnosis of any circumscribed retroperitoneal mass. These tumors can be successfully treated with surgery that leads to an excellent outcome, even in case of incomplete resections with tumor residuals $<2 \mathrm{~cm}$.
\end{abstract}

Key Words: Ganglioneuroma - Retroperitoneum • Children • Management.

\section{Introduction}

Ganglioneuromas (GNs) are rare (one per million population), typically slow-growing, benign tumors arising from the autonomic nervous system. GNs are composed of mature Schwann cells, ganglion cells and nerve fibers, arising from the sympathetic ganglia. The GNs are most commonly diagnosed in children, with the female sex predominance (1). The retroperitoneum is a common site for GNs, and approximately half of the reported cases are extra-adrenal (2). However, GN can arise in any sympathetic tissue, such as neck, posterior mediastinum, adrenal glands, retroperitoneum and pelvis (3).
These tumors are generally asymptomatic because they grow slowly and are usually detected incidentally at a late stage as a large mass (4). GNs may occasionally present with abdominal pain, palpation of an abdominal mass or patients may have symptoms related to adjacent organs compression. A minority of GNs exhibit metabolic activity caused by vasoactive intestinal peptides, catecholamines, and testosterone and present with hypertension, diarrhea, and virilization (5). Although the diagnosis of GN cannot be reliably established on radiological findings alone, magnetic resonance imaging (MRI) and computed tomography (CT) scanning are the preferred imaging methods to assess these tumors (6). A complete surgical removal 
is the treatment of choice whenever possible and it usually gives an excellent outcome (7). Unlike smaller GNs that are commonly diagnosed in childhood, large GNs tumors are mainly diagnosed after the age of ten. To date, only a few cases of giant extra-adrenal retroperitoneal GNs were reported in pediatric patients, especially below 10 years of age.

We present herein a case affecting a 9-year-old girl, discuss the therapeutic approach and briefly review literature on this rare pediatric condition.

\section{Case Report}

A 9-year-old girl, with a medical history of surgical correction of clubfoot three months earlier, presented to our department with an incidentally detected abdominal mass during diagnostic workup for orthopedic surgery. The physical examination revealed a painless palpable mass on the right side of her abdomen. Blood pressure was within the normal range. Family history was negative for neurofibromatosis and other cancer syndromes. Laboratory investigations (complete blood count, serum electrolytes, immunoglobulins, and urine) and endocrine tests (cortisol, adrenocorticotropic hormone levels, and 24-hour urinary catecholamines) were all within normal ranges. Routine tumor markers were also negative. Abdominal ultrasound examination revealed a right-sided extra-adrenal
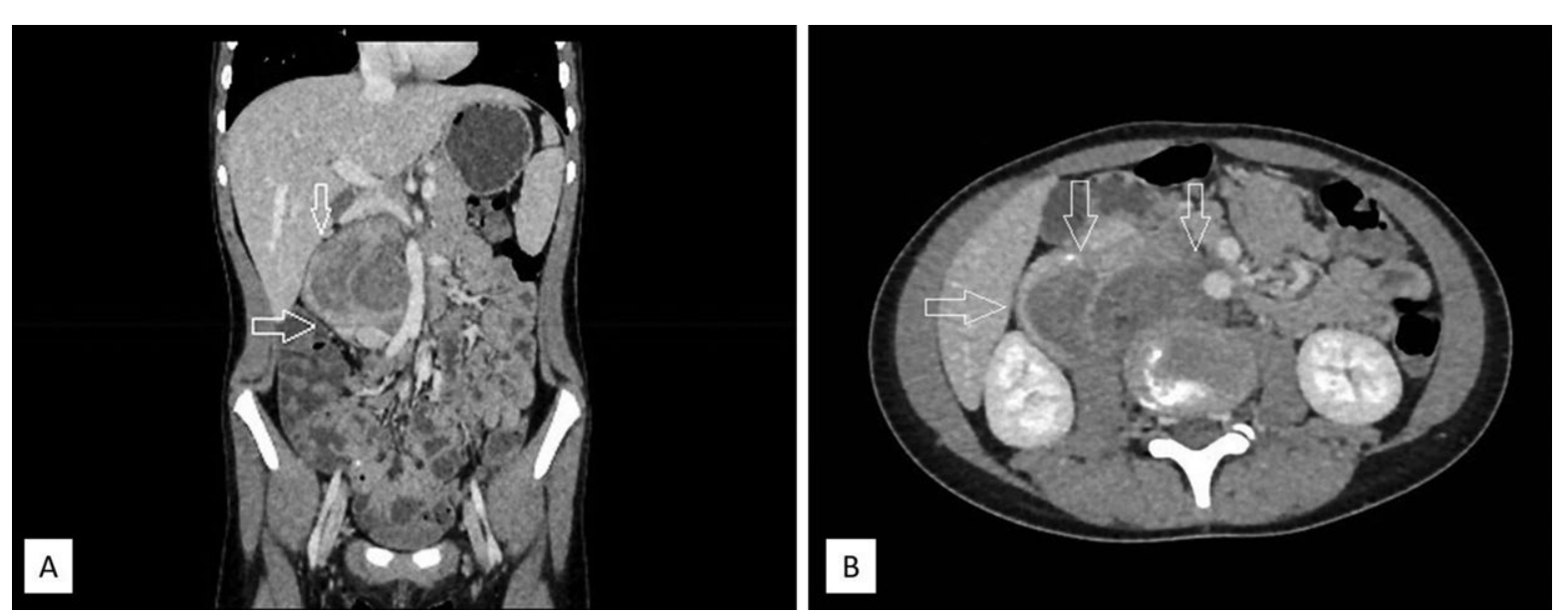

Fig. 1A-B. Contrast Enhanced Coronal (a) and Axial (b) CT scan Showing a Large Heterodense Mass in the Right Upper Abdomen (white Arrows), Compressing Inferior Vena Cava with Incomplete Encasing of Abdominal Aorta. hypoechogenic, somewhat heterogeneous solid mass, occupying the right aspect of the retroperitoneum, measuring $7 \mathrm{~cm}$ in its largest diameter.

Subsequent contrast-enhanced CT scan confirmed a vividly enhancing solid right-sided extraadrenal mass measuring $7.4 \times 6.6 \times 4.2 \mathrm{~cm}$ that was compressing/involving the infrahepatic part of inferior vena cava (IVC), right renal vein and artery with an incomplete encasing of the abdominal aorta (Fig. 1A-B). IVC was markedly dilated. A provisional diagnosis of non-functioning, probably benign, right extra-adrenal tumor was made. MRI was also performed to further characterize the mass (not shown). An ultrasonography-guided percutaneous fine-needle biopsy (FNAB) of the tumor was performed revealing a cellular neoplasm composed of ganglion cells and spindle-shaped cells with "cigar-shaped nuclei". Morphological findings indicated a ganglioneuroma (GN).

After obtaining parental consent, the patient underwent surgery. The lesion was approached transperitoneally through an extended transverse supra-umbilical incision. After mobilization of the right colon, an encapsulated right-sided extra-adrenal retroperitoneal tumor was carefully dissected away from the surrounding adherent structures including IVC, right renal vein and artery, abdominal aorta, and the right kidney, but patency of the compressed vessels were maintained (Fig. 2A-B). 

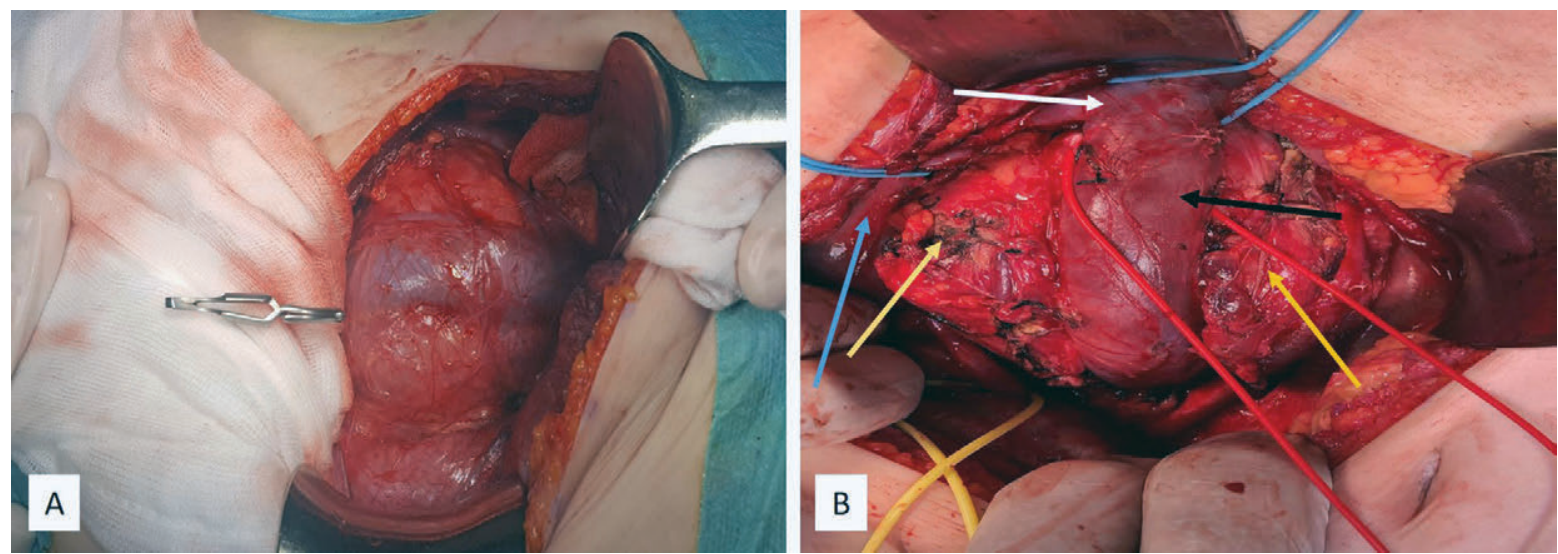

Fig. 2A-B. Intraoperative Images of the Tumor (yellow Arrows) Arising from Retroperitoneum That Was Adherent to the Infrahepatic Vena Cava (black Arrow), Right Renal Artery and Vein (white Arrow), and the Right Kidney before (blue Arrow) (a) and after Mobilization and Exposure of Large Blood Vessels (b).
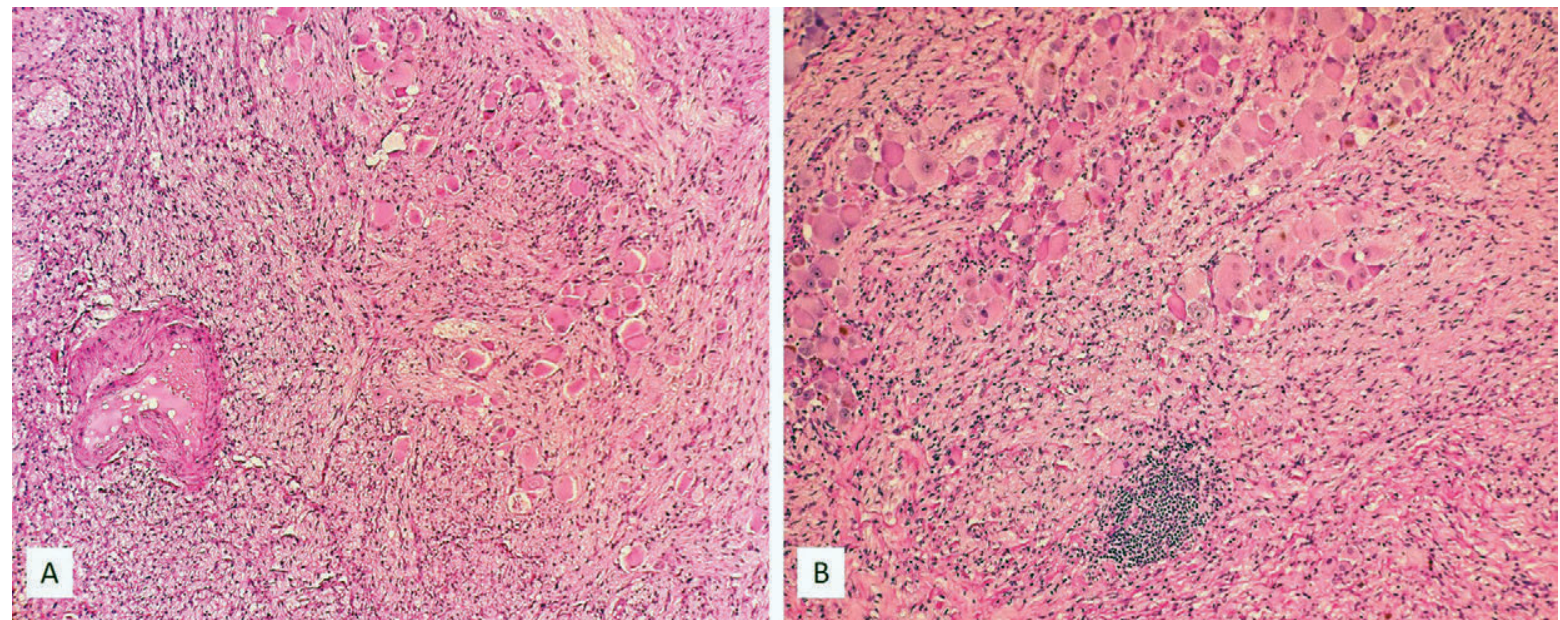

Fig. 3A-B. Hematoxylin and Eosin (HङE) Slides of the Moderately Cellular Neoplasm at Low Power (40') Magnification (a); the Tumor Was Composed of Admixture of Schwann Cells and Scattered Nests and Clusters of Ganglion Cells within the Fibrous Stroma. The Multinucleated Ganglion Cells Were Numerous Exhibiting Only Mild to Moderate Atypia (b) (100× Magnification).

Enlarged retroperitoneal lymph nodes were not detected. The small cuff of tumor capsule about 0.5 $\mathrm{cm}$ was left behind the fascia transversalis, as it was not possible to safely dissect it from the paravertebral space and the underlying spinal nerves. The tumor specimen was submitted to histopathology analysis that confirmed the mass to be a GN (Fig. 3A-B). The patient had an uneventful postoperative recovery and was discharged on the seventh day. One-year follow-up consisted of visits every 3 months revealing no recurrence of the tumor.

\section{Discussion}

Ganglioneuromas (GNs), well-differentiated, slowgrowing, usually non-secreting and asymptomatic benign tumors, belong to the group of neuroblastic tumors originating from neural crest cells that comprise a spectrum of both benign tumors like GN and malignant tumors such as (ganglio)neuroblastomas (8). Although GNs are often localized in the retroperitoneum, they account for only $1 \%$ of primary retroperitoneal tumors and approximately 
half of the reported cases are extra-adrenal (2). The differential diagnosis of solid retroperitoneal mass includes various tumors with neurogenic/neuroblastic origins (ganglioneuroma, ganglioneuroblastoma intermixed, neuroblastoma, neurofibroma, schwannoma, and pheochromocytoma), adrenal gland tumors (adrenocortical adenoma and carcinoma), various lymphomas (usually non-Hodgkin type), soft tissue sarcomas (e.g. liposarcoma), and germ cell tumors (e.g. teratoma) (9).

Extra-adrenal retroperitoneal ganglioneuromas in pediatric population are very rare. Our literature survey (PubMed/MEDLINE, Scopus, and Web of Science) revealed a few reported cases and small case series $(1,4,7,10-20)$ with very few cases reported in the patients $<10$ years (summarized in Table 1). Scherer et al. (2001) reported the largest case series of 5 patients ( 4 patients under the age of 10) depicting the radiological (CT and MRI) features of retroperitoneal GNs. The tumor size varied between 4 and $11 \mathrm{~cm}$ and girls were predominantly affected (4/5). Although GNs may present as an isolated finding as confirmed in our case, these tumors may be occasionally seen in association with neurofibromatosis type 1 (NF1) (21-23).

The widespread use of imaging techniques in clinical practice has contributed to an increase in the number of GNs detected incidentally. However, it is difficult to make an accurate preoperative diagnosis of GN and definitive diagnosis is based on histopathological analysis after surgical excision of the tumor. In our case, the preoperative diagnosis of GN was obtained by FNAB and was further confirmed by histopathological analysis. In the current literature, reports of GN that are reliably diagnosed by FNAB and its cytological appearance are scarce. A literature search revealed only a few cases diagnosed by FNAB at this location (24, 25). GNs on FNAB can be difficult to differentiate from other neurogenic/neuroblastic tumors, peripheral nerve sheath tumors, such as schwannomas or neurofibromas and a meticulous search for mature ganglion cells is critical for making an accurate diagnosis. Taken together, a broad differential diagnosis and subtle morphological details give FNAB a limited clinical utility (26).

Retroperitoneal GNs may clinically remain silent for a long time and the diagnosis is often incidental. Despite their benign nature, retroperitoneal GNs show the tendency to partially or completely surround large blood vessels without compromising the lumen in most cases, making their surgical excision extremely challenging (27).

The optimal treatment approach for most patients with retroperitoneal GN is a complete surgical excision (7). However, this approach has certain limitations, particularly in the case in which the large GN surrounds and compresses large blood vessels making its dissection extremely difficult. A mitigating circumstance is a fact that the enclosed blood vessels commonly have no narrow lumen or filling defect (28). In this case, GN enclosed IVC

$\begin{aligned} & \text { Table 1. A Summary of the Previous Studies that Reported Extra-adrenal Retroperitoneal Ganglioneuromas in Children } \\
& <10 \text { Years }\end{aligned}$
\begin{tabular}{llll}
\hline References (year) & Age of patients & & Tumor size \\
\hline Menon et al. (2020) (10) & 8 & 5.1 & Location $^{\ddagger}$ \\
\hline Maher et al. (2014) (17) & 3 & Not available & Retroperitoneum $^{\S}$ \\
\hline Noguchi et al. (2010) (18) & 2.6 & Not available & Retroperitoneum $^{\S}$ \\
\hline Zugor et al. (2009) (19) & $5($ case\#1); 8 (case\#2) & 5.2 (case\#1); 5.6 (case\#2) & Retroperitoneum $^{\S}$ \\
\hline Scherer et al. (2001) (4) & $5(4<10)$ & $4.1-11$ & Retroperitoneum $^{\S}$ \\
\hline Vara Castrodeza et al. (2000) (20) & 4 & 5 & Retroperitoneum $^{\S}$ \\
\hline Jain et al. (1999) (14) & 5 & Not available & Retroperitoneum $^{\S}$ \\
\hline Lin et al. (1997) (15) & 5 & Not available & Retroperitoneum $^{\S}$ \\
\hline
\end{tabular}

"The literature survey included PubMed/MEDLINE, Scopus and Web of Science databases using the following keywords: "Ganglioneuroma", "Retroperitoneum", "Extra-adrenal", "Children", and "Pediatrics". The studies were then filtered based on the patients' age ( $<10$ years) and anatomic location (retroperitoneal but extra-adrenal); ${ }^{\dagger}$ Years; ${ }^{\dagger}$ Largest diameter $(\mathrm{cm}) ;{ }^{\circledR}$ Extra-adrenal. 
and right renal artery and vein and compressed the anterior side of the right kidney. The dissection of GN away from IVC was the crucial step in the surgery. In our case, all large blood vessels were protected effectively.

Although the postoperative prognosis is usually excellent, long-term follow-up is recommended because of sporadically reported cases of local recurrences (29). However, Decarolis et al. suggested that even incomplete resection of GN is not associated with increased risk of progression if the tumor residuals are $<2 \mathrm{~cm}$ (29). Following the suggestion of other authors, we also performed a subtotal resection without endangering adjacent vital structures. This approach appears to be sufficient for the successful treatment of GN (30) as the disease did not recur within one-year follow-up period.

\section{Conclusions}

Although pediatric extra-adrenal retroperitoneal GNs are rare, their propensity for enveloping major blood vessels is not. GNs should be considered in the differential diagnosis of any circumscribed retroperitoneal mass in pediatric population.

Authors' Contributions: Conception and design: ZZ and SV; Acquisition, analysis and interpretation of data: ZZ, NR, ISP, FS and SV; Drafting the article: ZZ and SV; Revising the article critically for intellectual content: ZZ, NR, ISP, FS, and SV; Approved final version of the manuscript: ZZ, NR, ISP, FS, and SV.

Conflict of Interest: The authors declare no conflict of interest.

Research Ethics and Patient's Consent: The study was conducted according to the World Medical Association Declaration of Helsinki. The authors appreciate the patient's family for consenting to the report of this instructive case.

\section{References}

1. Esen HK, Esen O, Irsi C. Retroperitoneal Ganglioneuroma: Mimicking an ovarian mass in a child. Pak J Med Sci. 2015;31(3):724-6.

2. Cocieru A, Saldinger PF. Images in surgery: retroperitoneal ganglioneuroma. Am J Surg. 2011;201(1):e3-4.

3. Spinelli C, Rossi L, Barbetta A, Ugolini C, Strambi S. Incidental ganglioneuromas: a presentation of 14 surgical cases and literature review. Journal of endocrinological investigation. 2015;38(5):547-54.

4. Scherer A, Niehues T, Engelbrecht V, Modder U. Imaging diagnosis of retroperitoneal ganglioneuroma in childhood. Pediatr Radiol. 2001;31(2):106-10.

5. Yamaguchi K, Hara I, Takeda M, Tanaka K, Yamada Y, Fujisawa M, et al. Two cases of ganglioneuroma. Urology. 2006;67(3):622 e1-4.

6. Guo YK, Yang ZG, Li Y, Deng YP, Ma ES, Min PQ, et al. Uncommon adrenal masses: CT and MRI features with histopathologic correlation. Eur J Radiol. 2007;62(3):35970 .

7. Arab N, Alharbi A. Retroperitoneal Ganglioneuroma (GN): Case report in 14 years old boy. Int J Surg Case Rep. 2019;60:130-2.

8. Zvizdic Z, Haxhija E, Chikha A, Milisic E, Jonuzi A, Vranic S. Laparoscopic Extirpation of Adrenal Gland Ganglioneuroma Incidentally Diagnosed during Evaluation for Patchy Alopecia Areata in an Adolescent Boy. Med Princ Pract. 2019;28(5):485-9.

9. Scali EP, Chandler TM, Heffernan EJ, Coyle J, Harris AC, Chang SD. Primary retroperitoneal masses: what is the differential diagnosis? Abdom Imaging. 2015;40(6):1887-903.

10. Menon J, Kumar A, Vaiphei K, Lal S. An interesting cause of chronic abdominal pain in a child. Trop Doct. 2020;50(1):63-4.

11. Urso DL, Vincenzo D, Curia A, Formaro C, Sicilia S. [Retroperitoneal ganglioneuroma: a case report]. Recenti Prog Med. 2008;99(11):559-60.

12. Mut DT, Orhan Soylemez UP, Demir M, Tanik C, Ozer A. Diagnostic imaging findings of pelvic retroperitoneal ganglioneuroma in a child: a case report with the emphasis on initial ultrasound findings. Med Ultrason. 2016;18(1):120-2.

13. Oue T, Yoneda A, Sasaki T, Tani G, Fukuzawa M. Total laparoscopic excision of retroperitoneal ganglioneuroma using the hanging method and a vessel-sealing device. J Laparoendosc Adv Surg Tech A. 2008;18(5):779-82.

14. Jain M, Shubha BS, Sethi S, Banga V, Bagga D. Retroperitoneal ganglioneuroma: report of a case diagnosed by fineneedle aspiration cytology, with review of the literature. Diagn Cytopathol. 1999;21(3):194-6.

15. Lin HC, Lu WT, Sheih CP, Liao YJ, Tseng SH, Li YW. A giant retroperitoneal ganglioneuroma with intraspinal involvement: report of one case. Zhonghua Min Guo Xiao Er Ke Yi Xue Hui Za Zhi. 1997;38(5):390-2.

16. Steinberg SH. Presacral retroperitoneal ganglioneuroma in a 4 1/2-year-old child; report of a case and review of the literature. J Pediatr. 1955;46(5):562-72. 
17. Maher OM, Marco SA, Sadanandan S, Fireman F, Sedrak A. Retroperitoneal ganglioneuroma and reversible posterior leukoencephalopathy in a child with acute lymphoblastic leukemia. J Pediatr Hematol Oncol. 2014;36(8):665-6.

18. Noguchi S, Kaga Y, Takahashi Y, Aoyagi K, Nakamura K, Kamiya Y, et al. [A case of recurrent paraneoplastic cerebellar ataxia with antibodies to GluR epsilon 2 causally related to ganglioneuroma]. No To Hattatsu. 2010;42(4):297301 .

19. Zugor V, Schott GE, Kuhn R, Labanaris AP. Retroperitoneal ganglioneuroma in childhood--a presentation of two cases. Pediatr Neonatol. 2009;50(4):173-6.

20. Vara Castrodeza A, Madrigal Rubiales B, Perez del Rio MJ, Garcia Hernandez JB, Ablanedo Ablanedo P. [Retroperitoneal ganglioneuroma in childhood]. Arch Esp Urol. 2000;53(7):648-51.

21. Bacci C, Sestini R, Ammannati F, Bianchini E, Palladino T, Carella M, et al. Multiple spinal ganglioneuromas in a patient harboring a pathogenic NF1 mutation. Clinical genetics. 2010;77(3):293-7.

22. Kannu P, Nour M, Irving M, Xie J, Loder D, Lai J, et al. Paraspinal ganglioneuroma in the proband of a large family with mild cutaneous manifestations of NF1, carrying a deep NF1 intronic mutation. Clinical genetics. 2013;83(2):191-4.

23. Kyoshima K, Sakai K, Kanaji M, Oikawa S, Kobayashi S, Sato A, et al. Symmetric dumbbell ganglioneuromas of bilateral C2 and C3 roots with intradural extension associated with von Recklinghausen's disease: case report. Surgical neurology. 2004;61(5):468-73; discussion 73.
24. Ponce-Camacho MA, Diaz de Leon-Medina R, MirandaMaldonado I, Garza-Guajardo R, Hernandez-Salazar J, Barboza-Quintana O. A 5-year-old girl with a congenital ganglioneuroma diagnosed by fine needle aspiration biopsy: a case report. CytoJournal. 2008;5:5.

25. Chiaffarano J, Alexander M, Yee-Chang M, Simsir A, Shi Y. Ganglioneuroma: An Unusual Entity Diagnosed on Endoscopic Ultrasound Guided Fine Needle Aspiration Biopsy. American journal of clinical pathology. 2016;146(suppl_1).

26. Ma J, Liang L, Liu H. Multiple cervical ganglioneuroma: A case report and review of the literature. Oncology letters. 2012;4(3):509-12.

27. Vasiliadis K, Papavasiliou C, Fachiridis D, Pervana S, Michaelides M, Kiranou M, et al. Retroperitoneal extraadrenal ganglioneuroma involving the infrahepatic inferior vena cava, celiac axis and superior mesenteric artery: A case report. Int J Surg Case Rep. 2012;3(11):541-3.

28. Zheng X, Luo L, Han FG. Cause of postprandial vomiting - a giant retroperitoneal ganglioneuroma enclosing large blood vessels: A case report. World J Clin Cases. 2019;7(17):2617-22

29. Decarolis B, Simon T, Krug B, Leuschner I, Vokuhl C, Kaatsch P, et al. Treatment and outcome of Ganglioneuro$\mathrm{ma}$ and Ganglioneuroblastoma intermixed. BMC Cancer. 2016;16:542.

30. De Bernardi B, Gambini C, Haupt R, Granata C, Rizzo A, Conte $\mathrm{M}$, et al. Retrospective study of childhood ganglioneuroma. J Clin Oncol. 2008;26(10):1710-6. 